\title{
Power optimization for multiple QoS, delay, and BER classes relying on finite-delay information theory
}

\author{
Chen Gong ${ }^{1}$, Qian $\mathrm{Gao}^{1}$, Lajos Hanzo ${ }^{3}$, Zhengyuan $\mathrm{Xu}^{1,2 *}$ \\ 1. Key Laboratory of Wireless-Optical Communications, Chinese Academy of Sciences, School of \\ Information Science and Technology, University of Science and Technology of China, Hefei 230027, China \\ 2. Shenzhen Graduate School, Tsinghua University, Shenzhen 518055, China \\ 3. School of Electronics and Computer Science, University of Southampton, Southampton, SO17 1BJ, U.K. \\ *Corresponding author, email: xuzy@ustc.edu.cn
}

\begin{abstract}
Future communication systems will include different types of messages requiring different transmission rates, packet lengths, and service qualities. We address the power-optimization issues of communication systems conveying multiple message types based on finite-delay information theory. Given both the normalized transmission rate and the packet length of a system, the actual residual decoding error rate is a function of the transmission power. We propose a generalized power allocation framework for multiple message types. Two different optimization cost functions are adopted: the number of service-quality violations encountered and the sum log ratio of the residual decoding error rate. We provide the optimal analytical solution for the former cost function and a heuristic solution based on a genetic algorithm for the latter one. Finally, the performance of the proposed solutions are evaluated numerically.
\end{abstract}

Keywords: 5G communication, short packet, ultra-dense, delay-limited, finite-length information theory, power optimization

Citation: C. Gong, Q. Gao, L. Hanzo, et al. Power optimization for multiple QoS, delay, and BER classes relying on finite-delay information theory [J]. Journal of communications and information networks, 2017, 2(1): $33-40$.

\section{Introduction}

The communication systems of the future are expected to support a wide variety of services, such as speech, instant messaging, image transfer, flawless $3 \mathrm{D}$ video, and more. The message rates, packet lengths, and service qualities required vary according to service types ${ }^{[1-5]}$. Both speech communication and instant messaging require short packets to guarantee a low delay, while image and video communication requires long packets for guaranteeing sufficiently low residual BER (Bit Error Ratio). Being able to handle so many different message types ${ }^{[6,7]}$ while simultaneously supporting different packet lengths and maintaining a high quality of services ${ }^{[8]}$ poses significant challenges for future ultra-dense communication systems.

For short packets, the Shannonian transmission

Manuscript received Dec. 20, 2016; accepted Feb. 8, 2017

This work is supported by the National Key Basic Research Program of China (No. 2013CB329201), Key Program of National Natural Science Foundation of China (No. 61631018), Key Research Program of Frontier Sciences of CAS (No. QYZDYSSW-JSC003), Key Project in Science and Technology of Guangdong Province (No. 2014B010119001), Shenzhen Peacock Plan (No. 1108170036003286), and the Fundamental Research Funds for the Central Universities. 
capacity conceived for block-lengths approaching infinity fails to describe the true communication performance adequately. To describe the actual transmission performance more accurately, we adopt finite-length information theory, which characterizes the transmission performance of finite packet lengths. The constrained capacity of delay-limited communications and the performance of finite-length block codes were studied in Refs. [9] and [10], respectively. Recently, Polyanskiy et al. carried out a comprehensive study of the attainable coding performance in the finite-length regime ${ }^{[11]}$, demonstrating that finite-length analysis does indeed provide more accurate results.

Existing treatises on the optimization of ultradense communication scenarios are mainly focused on the infinite-delay capacity, which is capable of accurately predicting the performance of long-packet services $^{[12-15]}$. The relationship between power and bandwidth-efficiency was investigated ${ }^{[16]}$. However, it is more realistic to investigate the system optimization of a wider range of services, including low-delay scenarios.

Against this background, our new contribution is that we provide the finite-length analysis of communication systems supporting multiple service types. Each service type is characterized by the transmission rate, the packet length and the required service qualities quantified by the residual DEP (Decoding Error Probability). Note that given the transmission rate and packet length, the residual DEP is a function of the transmission power. Hence, the transmission power allocation to each service type needs to be optimized to maximally increase the system's utility. More explicitly, we focus our attention on the power optimization for different service types encountered and propose a generalized framework, which is further refined for Gaussian channels. Two pertinent CFs (Cost Functions) are optimized: the number of service-quality violations and the sum $\log$ ratio of the residual DEP. The performances of the proposed solutions are characterized by our numerical results.

The remainder of this paper is organized as follows. In section 2 , we provide a communication system model for different message types associated with different transmission rates, packet lengths and qualities of service required. In section 3 , we formulate the power optimization problem of the system under consideration. In section 4, we provide the solution to the specific power optimization problem, while numerical results are provided in section 5 . Finally, section 6 concludes the paper.

\section{The system model}

\subsection{A service model with finite packet length}

Let us now consider a set of transceiver pairs associated with multiple service types, which are deployed in a certain high traffic-density area. Let $N$ be the number of transceiver pairs. For the transceiver pair $i(1 \leqslant i \leqslant N)$, let $n_{i}$ denote the packet length, $C_{i}$ denote the capacity of the transmission link, $R_{i}$ represent the message rate for receiver $i$, and $\epsilon_{i}$ denote the desired DEP. Furthermore, let $P_{i}$ denote the transmission power and $h_{i}$ denote the link gain for transceiver pair $i$. Again, the link capacity for infinite packet lengths is a function of the transmission power $P_{i}$, which is formulated as $C_{i}\left(P_{i}\right)$. The system configuration of multiple transceiver pairs associated with various service types is shown in Fig. 1.

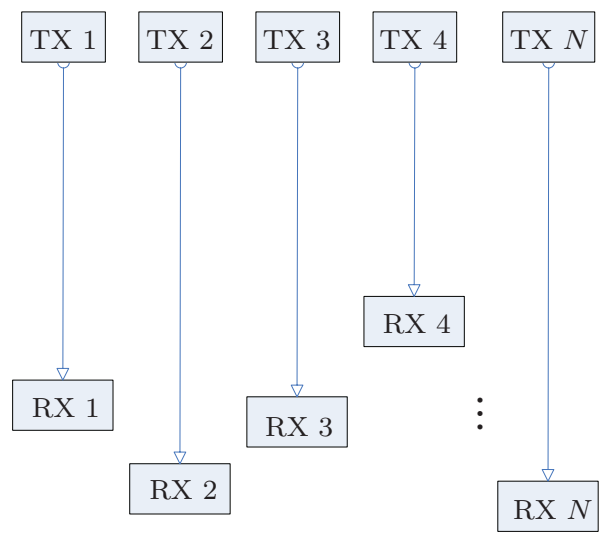

Figure 1 Communication system with multiple transceiver pairs for multiple message types

In the system under consideration, short to medium packet lengths are assumed, as exemplified 
by speech communication systems associated with short frame lengths and the IoT (Internet of Things). For short to medium packet lengths, there is a significant gap in infinite-length information theory. More specifically, we have the following approximation of the achievable transmission rate $R_{i}$ for the block length $n_{i}$ and the desirable DEP $\epsilon_{i}{ }^{[11]}$,

$$
\begin{aligned}
n_{i} R_{i} \cong & n_{i} C_{i}\left(\left|h_{i}\right|^{2} P_{i}\right)-\sqrt{n_{i} V_{i}\left(\left|h_{i}\right|^{2} P_{i}\right)} Q^{-1}\left(\delta_{i}\right) \\
& +\frac{1}{2} \operatorname{lb} n_{i},
\end{aligned}
$$

where $V_{i}\left(P_{i}\right)$ denotes the channel's dispersion for the link of receiver $i$ at transmission power $P_{i}$. The channel's dispersion is characterized by the variance of information density $\mathrm{lb} \frac{p(x, y)}{p(x) p(y)}$ with respect to the channel's input-output pair $(x, y)$. For the power allocation in this study we assume equality for the approximation above in Eq. (1) to obtain the DEP at transmission power $P_{i}$. Different CFs can be obtained based on the actual DEP $\delta_{i}$ and the desired DEP $\epsilon_{i}$.

\subsection{Power constraints}

We consider the sum of the individual transmission power constraints, assuming that $\sum_{i=1}^{N} P_{i}$ does not exceed a preset maximum of $P_{m}$, formulated as

$$
\sum_{i=1}^{N} P_{i} \leqslant P_{m}
$$

Note that the optimal cost is a function of the maximum sum power $P_{m}$. In section 5 , we will characterize the system performance with respect to the sum-power $P_{m}$, and compare it to that of the benchmark.

\section{Problem formulation}

We aim to allocate the power transmitted to different receivers, based on the sum-power constraint. The $\mathrm{CF}$ to be minimized can be formulated in different ways. In the remainder of this section, we will first outline different forms of the $\mathrm{CF}$ and provide a general framework for the power allocation. Then, we will refine the formulations for the Gaussian channel.

\subsection{General optimization framework}

We provide the following two forms of the CFs, each of which may lead to different optimization problem objectives.

1. The number of service quality violations. The $\mathrm{CF}$ for each receiver is given by $\mathbf{1}\left(\delta_{i}>\epsilon_{i}\right)$, which represents the indicator function concerning whether or not the actual DEP $\delta_{i}$ exceeds the required DEP $\epsilon_{i}$. The total cost for the $N$ pairs is then formulated as $\sum_{i=1}^{N} \mathbf{1}\left(\delta_{i}>\epsilon_{i}\right)$, which is quantified as the number of service quality violations.

2. The sum $\log$ ratio. The sum log ratio reflects the fact that the true DEP $\delta_{i}$ may exceed the desired DEP $\epsilon_{i}$. The CF for each receiver $i$ is given by $\max \left(0, \log \frac{\delta_{i}}{\epsilon_{i}}\right)$, which implies zero cost for $\delta_{i} \leqslant \epsilon_{i}$. The total cost for all $N$ transceiver pairs is given by $\sum_{i=1}^{N} \max \left(0, \log \frac{\delta_{i}}{\epsilon_{i}}\right)$.

In our problem formulation, we aim for minimizing each $\mathrm{CF}$ for the set of all $N$ receivers, subject to the sum-power constraint. We assume equality as the relationship between the DEP and the transmission power in Eq. (1). Let $W_{i}\left(\delta_{i}, \epsilon_{i}\right)$ denote the $\mathrm{CF}$ of receiver $i$, which can be either the number of service quality violations or the sum log ratio. The optimization problem can then be formulated as follows,

$$
\begin{aligned}
\min _{\left\{P_{i}\right\}_{i=1}^{N}} & \sum_{i=1}^{N} W_{i}\left(\delta_{i}, \epsilon_{i}\right) \text { s.t. } \\
n_{i} R_{i} & \leqslant n_{i} C_{i}\left(\left|h_{i}\right|^{2} P_{i}\right)-\sqrt{n_{i} V_{i}\left(\left|h_{i}\right|^{2} P_{i}\right)} Q^{-1}\left(\delta_{i}\right) \\
& +\frac{1}{2} \operatorname{lb} n_{i} ; \\
\sum_{i=1}^{N} P_{i} & \leqslant P_{m} .
\end{aligned}
$$

Note that in the above optimization framework, neither the capacity $C_{i}(\cdot)$ of the infinite-length regime nor the dispersion $V_{i}(\cdot)$ has been specified, since they assume different channels are in use. The inequality in Eq. (3) for finite-length performance stemming from the approximation in Eq. (1) is valid. Let us now consider the Gaussian channel and specify the optimization problem shown in Eq. (3). 


\subsection{Optimization for Gaussian channels}

The Gaussian channel is commonly adopted for quantifying the performance of power allocation, since the capacity and the channel dispersion can be expressed in tractable forms. Thus, in addition to the general optimization framework, we refine the power allocation problem formulation for Gaussian channels.

For Gaussian channels, assuming that the additive Gaussian noise power is given by $\sigma_{i}^{2}$ for the receiver associated with message type $i$, we have the following infinite-length capacity,

$$
C_{i}\left(\left|h_{i}\right|^{2} P_{i}\right)=\frac{1}{2} \log \left(1+\frac{\left|h_{i}\right|^{2} P_{i}}{\sigma_{i}^{2}}\right) .
$$

Additionally, the channel's dispersion function can be expressed according to Ref. [11], as

$$
V_{i}\left(\left|h_{i}\right|^{2} P_{i}\right)=\frac{\left|h_{i}\right|^{2} P_{i}\left(\left|h_{i}\right|^{2} P_{i}+2\right)}{2\left(\left|h_{i}\right|^{2} P_{i}+1\right)^{2}} \mathrm{lb} e .
$$

Based on the general optimization problem framework, the optimization problem for Gaussian channels is given as follows,

$$
\begin{aligned}
& \min _{\left\{P_{i}\right\}_{i=1}^{N}} \sum_{i=1}^{N} W_{i}\left(\delta_{i}, \epsilon_{i}\right) \\
& \text { s.t. } \quad R_{i}=\frac{1}{2} \log \left(1+\frac{\left|h_{i}\right|^{2} P_{i}}{\sigma_{i}^{2}}\right)
\end{aligned}
$$

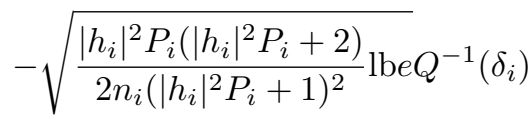

$$
\begin{aligned}
& +\frac{1}{2} \frac{\mathrm{lb} n_{i}}{n_{i}} \text {; } \\
& \sum_{i=1}^{N} P_{i} \leqslant P_{m} .
\end{aligned}
$$

Note that the convexity of the optimization problems (3) and (6) is difficult to ensure due to the specific form of the CF $W_{i}\left(\delta_{i}, \epsilon_{i}\right)$, as exemplified by the number of service quality violations or the sum-logratio. Thus, a general solution based on solving a convex optimization problem is difficult to obtain. Hence, we provide solutions to the above-mentioned pair of CFs. The details are outlined in the remainder of this paper.
The optimization can be specified based on different forms of both the infinite-length capacity $C_{i}$ and the dispersion $V_{i}$ for different types of non-Gaussian channels. Other types of channels, such as the binary symmetric channel, the erasure channel, and channels with signal-dependent Gaussian shot noise, can also be readily considered. Parameters other than transmission powers readily be optimized within our framework.

\section{Problem solution}

Let us now focus our attention on solving the optimization problems (c.f. Eqs. (3) and (6)) for the above pair of CFs $W_{i}\left(\delta_{i}, \epsilon_{i}\right)$. For the number of service quality violations, we provide an analytical solution to the general optimization framework; and for the sum log ratio, we provide numerical solutions for the classic Gaussian channel.

\subsection{Minimizing the number of services- quality violations}

For receiver $i$, let $\hat{P}_{i}$ denote the minimum transmission power such that the residual DEP is lower than or equal to $\epsilon_{i}$, (i.e., the service quality required for receiver $i$ is satisfied). The $\mathrm{CF}$ can be minimized by adjusting the transmission power $P_{i}$ appropriately.

Without a loss of generality, we assume $\hat{P}_{1} \leqslant \hat{P}_{2} \leqslant$ $\cdots \leqslant \hat{P}_{N}$. Then we arrive at the optimal solutions to the general optimization framework (c.f. Eq. (3)).

Theorem 1 The optimal solution to the general framework (c.f. Eq. (3)) is given by letting $P_{i}=\hat{P}_{i}$ for $i \leqslant M_{0}$ and letting $P_{i}>\hat{P}_{i}$ for $i>M_{0}$, where the threshold $M_{0}$ is determined as follows,

$$
M_{0}=\max \left\{M: \sum_{i=1}^{M} \hat{P}_{i} \leqslant P_{m}\right\} .
$$

Proof Consider any other power allocation $\left\{\tilde{P}_{i}\right\}_{i=1}^{N}$. We count the number of indices $i$ such that $\tilde{P}_{i}<\hat{P}_{i}$. Upon using $\mathbb{S}$ to denote the set of indices $i$ such that $\tilde{P}_{i}<\hat{P}_{i}$, we have

$$
\sum_{i \in \mathbb{S}^{c}} \hat{P}_{i} \leqslant \sum_{i \in \mathbb{S}^{c}} \tilde{P}_{i} \leqslant P_{m} .
$$


Since $\hat{P}_{i}$ increases with $i$, we have

$$
\sum_{i=1}^{\left|\mathbb{S}^{c}\right|} \hat{P}_{i} \leqslant \sum_{i \in \mathbb{S}^{c}} \hat{P}_{i}<P_{m} .
$$

Then, according to the definition of $M_{0}$ (c.f. Eq. (7)), we have $\left|\mathbb{S}^{c}\right| \leqslant M_{0}$ and $|\mathbb{S}| \geqslant N-M_{0}$. Based on the definition of $\mathbb{S}$, for the power allocation $\left\{\tilde{P}_{i}\right\}$ we have

$$
\sum_{i=1}^{N} \mathbf{1}\left(\delta_{i}>\epsilon_{i}\right) \geqslant \sum_{i=1}^{N} \mathbf{1}\left(\tilde{P}_{i}<\hat{P}_{i}\right)=|\mathbb{S}| \geqslant N-M_{0} .
$$

Thus the CF cannot be smaller than the one given by the power allocation $\left\{\hat{P}_{i}\right\}_{i=1}^{N}$.

For the Gaussian channel we can further specify the transmission power $\hat{P}_{i}$ in Eq. (11). The optimal power allocation is given by Theorem 1 , while the minimum cost is given by $\left(N-M_{0}\right)$.

$$
\begin{aligned}
\hat{P}_{i}= & \min \left\{P_{i}: R_{i} \leqslant \frac{1}{2} \log \left(1+\frac{\left|h_{i}\right|^{2} P_{i}}{\sigma_{i}^{2}}\right)\right. \\
& -\sqrt{\frac{\left|h_{i}\right|^{2} P_{i}\left(\left|h_{i}\right|^{2} P_{i}+2\right)}{2 n_{i}\left(\left|h_{i}\right|^{2} P_{i}+1\right)^{2}} \operatorname{lbe} Q^{-1}\left(\epsilon_{i}\right)} \\
& \left.+\frac{1}{2} \frac{\mathrm{lb} n_{i}}{n_{i}}\right\} .
\end{aligned}
$$

\subsection{Maximizing the sum log ratio}

Since the convexity of the constraints is difficult to prove, for the sum log ratio $\mathrm{CF}$ we resort to a numerical solution using a GA (Genetic Algorithm).

Specifically, the GA is applied to a group of power allocation vectors $\left\{P_{i}\right\}_{i=1}^{N}$. Let $\boldsymbol{P}^{(k)} \triangleq\left\{P_{i}^{(k)}\right\}_{i=1}^{N}$ denote the power vector of the individual $k$, and let $\boldsymbol{P} \triangleq\left\{\boldsymbol{P}^{(k)}: 1 \leqslant k \leqslant G\right\}$ denote the group of power vectors, where $G$ is the group size. Given the power $P_{i}^{(k)}$, let $\delta_{i}\left(P_{i}^{(k)}\right)$ denote the corresponding DEP according to Eq. (1), where equality is achieved. The $\mathrm{CF}$ for the power vector $\boldsymbol{P}^{(k)}$ is given by

$$
\mathcal{W}\left(\boldsymbol{P}^{(k)}\right) \triangleq \sum_{i=1}^{N} W_{i}\left(\delta_{i}\left(P_{i}^{(k)}\right), \epsilon_{i}\right) .
$$

After initializing the group $\boldsymbol{P}=\left\{\boldsymbol{P}^{(k)}: 1 \leqslant k \leqslant\right.$ $G\}$, the GA is invoked iteratively. Each iteration consists of discarding certain low-fitness individuals, the crossover of individuals with low cost and mutation to prevent premature convergence to a local optimum. In each iteration, the $\mathrm{CF} \mathcal{W}\left(\boldsymbol{P}^{(k)}\right)$ is calculated for each power vector individual $\boldsymbol{P}^{(k)}$. The worst half of the individuals are discarded, and replaced by those generated from the crossover of the better half. After the crossover, the transmission power is normalized to limit the sum-power to $P_{m}$ if it exceeds this value. A slight change of the power vector is then invoked as the mutation, where the power vector is again normalized to limit the sumpower to $P_{m}$ if it exceeds $P_{m}$. The GA process terminates after performing an affordable number of iterations, or when the CF falls below a preset threshold.

\section{$5 \quad$ Numerical results}

Consider the communication scenario of multiple services being provided to a densely populated cellular network for clustered receivers. The services can be modeled as a combination of five different packet lengths and two desired DEPs. The packet lengths are 100, 150, 200, 250, and 300; and for each packet length, the maximum DEPs can be either 0.005 or 0.01 . Furthermore, we consider two normalized throughputs of 0.5 and 1.0. Without a loss of generality, we assume that the additive noise variances are one for all types of receivers, and that all of the link gains are one as well. We then evaluate the performance of the proposed power allocation scheme and compare it to that of uniform power allocation.

We first plot the required power distribution for all message types, while ensuring that the desired DEP is satisfied. Fig. 2 shows the sorted transmission power, where the left ten indices represent the power required for a throughput of 0.5 , and the right ten indices represent the power required for a throughput of 1.0. As expected, the powers required for different combinations of packet lengths and DEPs are different. The gap between the maximum and minimum powers for the throughput 1.0 is larger than that of the throughput 0.5 . The different powers demonstrate the need to introduce finite-length information 
theory to the optimization of services associated with different packet lengths and different DEPs.

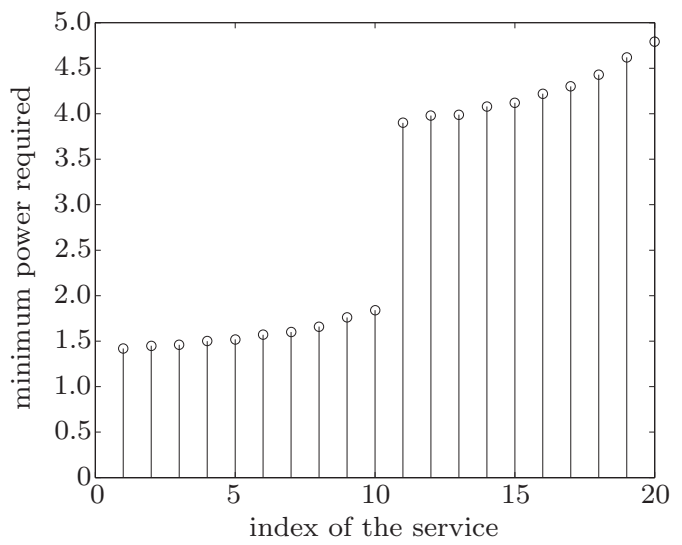

Figure 2 Distribution of required transmission power for different services

We next consider the $\mathrm{CF} \sum_{i=1}^{N} \mathbf{1}\left(\delta_{i}>\epsilon_{i}\right)$, and plot the number of adequately satisfied service requests of the proposed optimization approach for the throughputs of both 0.5 and 1.0 in Fig. 3. The number of adequately satisfied service requests recorded for uniform power allocation is employed as the comparison benchmark. As expected, the number of service quality violations was reduced for the proposed optimal power allocation.

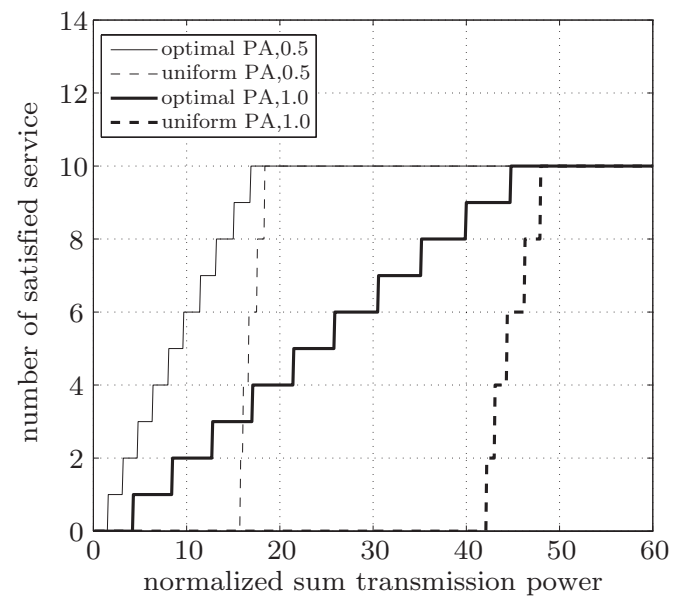

Figure 3 Number of satisfied service requests for the proposed optimal power allocation

Let us now consider the $\mathrm{CF} \sum_{i=1}^{N} \max \left(0, \log \frac{\delta_{i}}{\epsilon_{i}}\right)$, and plot it in Fig. 4 for both throughputs of 0.5 and 1.0. The GA has 1000 individuals, and the numbers of iterations are set to be 10,20,50, and 100. Again, uniform power allocation is adopted for benchmark- ing. It is apparent in Fig. 4 that the Genetic algorithm is capable of reducing the cost compared to that of uniform power allocation. Increasing the number of iterations can reduce the cost as well. In other words, different numbers of iterations comprise of a tradeoff between performance and complexity.

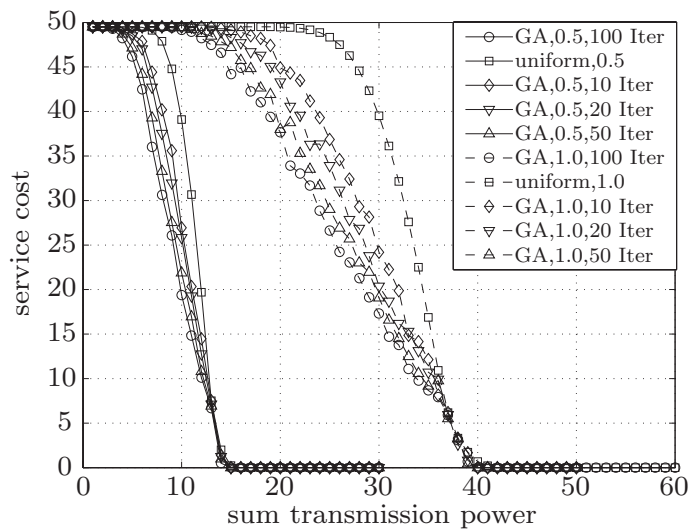

Figure 4 Service cost for the proposed optimal power allocation

\section{Conclusions}

We adopted finite-length information theory to characterize the performances of communication systems associated with multiple message types relying on short block lengths and considered different transmission rates, packet lengths and qualities of service required. We also formulated the poweroptimization problem for multiple message types and provided both analytical and numerical solutions. The performance of the proposed power allocation approach was evaluated numerically. Our future research studies may include further optimization of the transmission system based on diverse users and service behaviors, as well as on channels.

\section{References}

[1] 5G System [Z]. Ericsson White Paper, 2015.

[2] 5G Vision [Z]. DMC Research and Development Center. Samsung Electronics Co., Ltd., 2015.

[3] 5G radio access [Z]. Ericsson White Paper, 2015.

[4] Rethink mobile communications for 2020+ [Z]. Future Mobile Communication Forum White Paper, 2014.

[5] P. Smulders. The road to $100 \mathrm{Gbit} / \mathrm{s}$ wireless and beyond: basic issues and key directions [J]. IEEE Communications Magazine, 2013, 51(12): 86-91. 
[6] N. Bhushan, J. Y. Li, D. Malladi, et al. Network densification: the dominant theme for wireless evolution into 5G [J]. IEEE Communications Magazine, 2014, 52(2): 82-89.

[7] IWPC. Evolutionary and disruptive visions towards ultra high capacity networks, for crowd and broadband/dense applications access, backhaul and user equipment [Z]. White Paper, 2014.

[8] P. Lin, W. Chou, T. Lin. Achieving airtime fairness of delay sensitive applications in multirate IEEE 802.11 wireless LANs $[\mathrm{J}]$. IEEE Communications Magazine, 2013, 49(9): 169-175.

[9] J. Akhtman, R. Maunder, L. Hanzo. Constrained capacity of delay-limited wireless transceivers [C]//IEEE 70th Vehicular Technology Conference Fall, Anchorage, USA, 2009: 1-4.

[10] J. Akhtman, R. Maunder, N. Bonello. Closed-form approximation of maximum free distance for binary block codes [C]//IEEE 70th Vehicular Technology Conference Fall, Anchorage, USA, 2009: 1-3.

[11] Y. Polyanskiy, H. V. Poor, S. Verdu. Channel coding rate in the finite blocklength regime [J]. IEEE Transactions on Information Theory, 2012, 56(5): 2307-2359.

[12] B. Soret, K. I. Pedersen, N. T. K. Jorgensen, et al. Interference coordination for dense wireless networks [J]. IEEE Communications Magazine, 2015, 53(1): 102-109.

[13] S. F. Yunas, M. Valkama, J. Niemela. Spectral and energy efficiency of ultra-dense networks under different deployment strategies [J]. IEEE Communications Magazine, 2015, 53(1): 90-101.

[14] A. Asadi, V. Sciancalepore, V. Mancuso. On the efficient utilization of radio resources in extremely dense wireless networks [J]. IEEE Communications Magazine, 2015, 53(1): 126-132.

[15] Y. M. Shi, J. Zhang, B. O'Donoghue, et al. Largescale convex optimization for dense wireless cooperative networks [J]. IEEE Transactions on Signal Processing, 2015, 63(18): 4729-4743.

[16] J. Akhtman, L. Hanzo. Power versus bandwidthefficiency in wireless communications: the economic perspective [C]//IEEE 70th Vehicular Technology Conference Fall, Anchorage, USA, 2009: 1-5.

\section{About the authors}

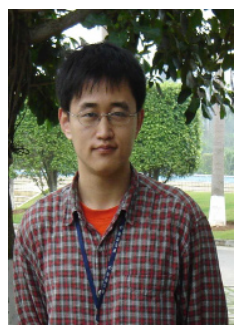

Chen Gong received the B.S. degree in electrical engineering and mathematics (minor) from Shanghai Jiao Tong University, Shanghai, China, in 2005, M.S. degree in electrical engineering from Tsinghua University, Beijing, China, in 2008, and Ph.D. degree in Columbia University, New York City, New York, USA, in 2012. He was a senior systems engineer in Qualcomm Re- search San Diego, San Diego, Qualcomm Inc., between Apr. 2012 and Dec. 2013. Now he is on the faculty of University of Science and Technology of China (USTC). His research interests fall into the general area of wireless communications, optical wireless communications, and signal processing. He has been selected by the "Young 1000 Talent Program" of China Government in 2014. (Email: cgong821@ustc.edu.cn)

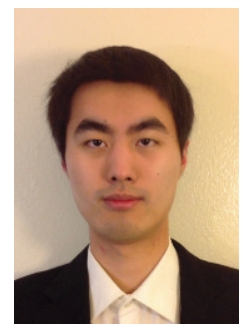

Qian Gao received an M.Sc. and a Ph.D. degree from University of California, Riverside in 2010 and 2014. He was with the Center of Ubiquitous Communications by Light from 2012 to 2014 and was a technical intern with the United Nations headquarters in summer 2010. He is currently on faculty list at the University of Science and Technology of China. He has (co)contributed multiple standard documents to IEEE 802.15 on visible light communications. His research interests include modulation and transceiver design for wireless/optical communications, and wireless full-duplex radio. He is a grant reviewer for OSA and National Natural Science Fund Committee of China, and is currently an associate editor of the EURASIP journal on wireless communications and networking. (Email: qgao@ustc.edu.cn)

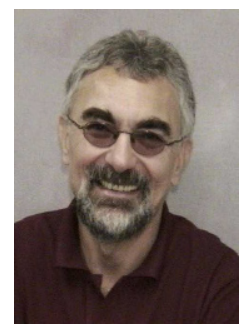

Lajos Hanzo, FREng, FIEEE, FIET, Fellow of EURASIP, DSc received his degree in electronics in 1976 and his doctorate in 1983. In 2009 he was awarded an honorary doctorate by the Technical University of Budapest and in 2015 by the University of Edinburgh. In 2016 he was admitted to the Hungarian Academy of Science. During his 40-year career in telecommunications he has held various research and academic posts in Hungary, Germany and the UK. Since 1986 he has been with the School of Electronics and Computer Science, University of Southampton, UK, where he holds the chair in telecommunications. He has successfully supervised $111 \mathrm{PhD}$ students, co-authored 20 John Wiley/IEEE Press books on mobile radio communications totalling in excess of 10000 pages, published more than 1600 research contributions at IEEE Xplore, acted both as TPC and General Chair of IEEE conferences, presented keynote lectures and has been awarded a number of distinctions. Currently he is directing a 60 -strong academic research team, working on a range of research projects in the field of wireless multimedia communications sponsored by industry, the Engineering and Physical Sciences Research Council (EPSRC) UK, the European Research Council's Advanced Fellow Grant and the Royal Society's Wolfson Research Merit Award. He is an enthusiastic supporter of industrial and academic liaison and he offers a range of industrial courses. He is also a Governor of the IEEE VTS. During 2008-2012 he was the Editor-in-Chief of the IEEE Press and a Chaired Pro- 
fessor also at Tsinghua University, Beijing. For further information on research in progress and associated publications please refer to http://www-mobile.ecs.soton.ac.uk Lajos has more than 25000 citations and an H-index of 60. (Email: lh@ecs.soton.ac.uk)

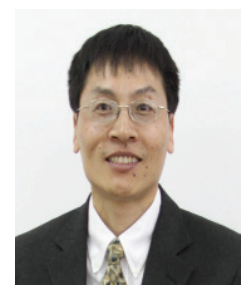

Zhengyuan Xu [Corresponding author] received his B.S. and M.S. degrees from Tsinghua University, China, and Ph.D. degree from Stevens Institute of Technology, USA. He was with University of California, Riverside, during 1999-2010, where he became a full professor with tenure and also a founding director of UC-Light Center. In 2010, he was selected by the "Thousand Talents Program" of China and appointed as a professor at Tsinghua University. Since 2013, he has been a professor in Department of Electronic Engineering and Information Science, School of Information Science and Technology, University of Science and Technology of China. He is founding director of Wireless-Optical Communications Key Laboratory of Chinese Academy of Sciences, and chief scientist of the National Key Basic Research Program of China. His research focuses on wireless communication and networking, optical wireless communications, geolocation, and signal processing. He has published over 260 journal and conference papers. He was associate editor and guest editor for different IEEE journals, and founding co-chair of IEEE GLOBECOM Workshop on Optical Wireless Communications. He has delivered tutorials, keynote speeches and invited talks on optical wireless communications in various international conferences. (Email: xuzy@ustc.edu.cn) 